\title{
On the Definition and the Approximate Continuity of the General Derivative.
}

\author{
By \\ Fumitomo MAEDA. \\ (Received Sept. 20, 1931)
}

In the previous paper ${ }^{(1)}$, I attempted to prove the fundamental theorem of the relation between the generalised integrals and their derivatives with respect to any set function $\phi(E)$, using the same definition of the general derivative as with respect to the Lebesgue measure, i.e. the general derivative of $\Phi(E)$, at a point $a$, with respect to $\phi(E)$ is the limit of $\frac{\Phi\left(M_{i}\right)}{\phi\left(M_{i}\right)}$ as $i$ becomes infinite, where $\left\{M_{i}\right\}$ is the $\phi$-regular sequence of point sets converging to the point $a$. I found that, except for the set of poles of $\phi(E)$, the same relations hold as in the case of the Lebesgue measure. To avoid this exception we must put certain restrictions on the definition of the general derivatives. Daniell $^{(2)}$ has recently given a new definition of the derivative and proved that the fundamental theorem holds without exception in Euclidian space. In this paper, I modify Daniell's definition so that $\left\{M_{i}\right\}$ is the $\phi$-regular monotone sequence of point sets, and I prove the fundamental theorem by a method involving the extension of Vitali's theorem which was discarded by Daniell. ${ }^{(3)}$

Next, under this definition of the general derivative, I define the approximate continuity of a point function with respect to the nonnegative set function $\phi(E)$, and prove that a necessary and sufficient condition, that a bounded $\phi$-measurable point function is a unique general derivative of a set function with respect to $\phi(E)$, is that the point function is approximately continuous with respect to $\phi(E)$.

\section{Definition of the General Derivatives.}

1. Let $\phi(E)$ be a completely additive function of normal sets in the Euclidian space $R_{k}$ of $k$ dimensions, and $\mathfrak{F}$ be a closed family of

(1) This journal, 1 (1931), 1-24.

(2) P. J. Daniell, Proc. London Math. Soc., (2), 30 (1930), 187-198.

(3) Ibid., 198. 
$\phi$-normal sets which contains all the Borel sets. Now assume that $\phi(E)$ is non-negative, since the case in which $\phi(E)$ has any sign can be easily reduced to that in which $\phi(E)$ is non-negative ${ }^{(1)}$.

Let $\lambda$ be a positive number greater than 1 , and $a$ be a point in the space $R_{k}$. If there exist a positive number $\kappa(a, \lambda)$ and a sequence of positive numbers $\left\{\rho_{i}\right\}$ converging to zero, so that

$$
\frac{\phi\left\{\bar{W}\left(a, \rho_{i}\right)\right\}}{\phi\left\{\bar{W}\left(a, \lambda \rho_{i}\right)\right\}} \geqq \kappa(a, \lambda)^{(2)} \quad(i=1,2, \ldots, n, \ldots)
$$

holds, then I say that the sequence of neighbourhoods $\left\{\bar{W}\left(a, \rho_{i}\right)\right\}$ is $\phi$-monotone.

Let $\left\{M_{i}\right\}$ be a sequence of the sets belonging to the family $\mathfrak{F}$. If a $\phi$-monotone sequence $\left\{\bar{W}\left(a, \rho_{i}\right)\right\}$ exists which satisfies the following inequalities

$$
\begin{aligned}
& M_{i} \leqq \bar{W}\left(a, \rho_{i}\right), \\
& \frac{\phi\left(M_{i}\right)}{\phi\left\{\bar{W}\left(a, \rho_{i}\right)\right\}} \geqq \vartheta(a), \quad(i=1,2, \ldots, n, \ldots)
\end{aligned}
$$

where $\vartheta(a)$ is a definite positive number which depends to the point $a$, then $\left\{M_{i}\right\}$ is called the $\phi$-regular monotone sequence of point sets which converges to the point $a$.

2. If, converging to a point $p$, there exists no $\phi$-regular monotone sequence of point sets for any value of $\lambda$ which is greater than 1 , then I say that the point $p$ is an essential pole ${ }^{(3)}$ of $\phi(E)$. In respect of the essential poles of $\phi(E)$ we have the following theorem :

The set of essential poles of $\phi(E)$ is of $\phi$-value zero.

This theorem has been proved by Daniell(4), but here I will give another proof.

Let $\dot{P}$ be the set of essential poles of $\phi(E)$ and $Q$ be the set of points, converging to which there exists no $\phi$-regular monotone sequence of point sets for $\lambda=2$. Then it is obvious that

$$
P \leqq Q,
$$

therefore, now it is sufficient to prove that $\phi(Q)=0$.

(1) Cf. my previous paper, 14.

(2) In this paper I use the same symbols as in the previous paper. cit., 188

(3) This point corresponds to that which Daniell called an irregular point. Loc.

(4) Loc. cit., 189. 
For the sake of brevity, it is assumed that the space is of two dimensions. Let $\gamma$ be a number greater than 2, then for a point $p$ of $Q$ there exists a positive number $\rho_{p}$, so that for any positive number $\rho<\rho_{p}$

$$
\frac{\phi\{\bar{W}(p, \rho)\}}{\phi .\{\bar{W}(p, 2 \rho)\}}<\frac{1}{\gamma} .
$$

Let $\alpha$ be a positive number and let $Q_{\alpha}$ denote the set of points of $Q$ where $2 \rho_{p}>\alpha$. Consider a net whose meshes are squares with sides of length $\alpha$. We take each of the meshes to be semi-closed, i.e. closed at the left, lower boundary, and open at the right, upper boundary. Let $W^{\prime}$ be a mesh which contains the point of $Q_{\alpha}$. Divide each side of $W^{\prime}$ into equal $2^{m}$ parts, then $W^{\prime}$ may be divided into $2^{m+1}$ squares. In these $2^{m+1}$ squares, let the squares which contain the point of $Q_{\alpha}$ be

$$
W_{1}^{\prime}, W_{2}^{\prime}, \ldots \ldots, W_{i}^{\prime}, \ldots, W_{k}^{\prime},
$$

where $k \leqq 2^{m+1}$.

Denote by $p_{i}$ the point of $Q_{\alpha}$ which is in $W_{i}^{\prime}$, then, since the side of $W_{i}^{\prime}$ is of length $\frac{\alpha}{2^{m}}$, it is obvious that

$$
W_{i}^{\prime}<\bar{W}\left(p_{i}, \frac{\alpha}{2^{m-1}}\right)
$$

therefore,

$$
\phi\left(W_{i}^{\prime}\right) \leqq \phi\left\{\bar{W}\left(p_{i}, \frac{\alpha}{2^{m-1}}\right)\right\}
$$

But by (1)

$$
\begin{aligned}
\phi\left\{\bar{W}\left(p_{i}, \frac{\alpha}{2^{m-1}}\right)\right\} & <\frac{1}{\gamma} \phi\left\{\bar{W}\left(p_{i}, \frac{\alpha}{2^{m-2}}\right)\right\} \\
& <\frac{1}{\gamma^{2}} \phi\left\{\bar{W}\left(p_{i}, \frac{\alpha}{2^{m-3}}\right)\right\} \\
& \cdots \ldots \ldots \\
& <\frac{1}{\gamma^{m-1}} \phi\left\{\bar{W}\left(p_{i}, \alpha\right)\right\} .
\end{aligned}
$$


Let $q$ be the centre of $W^{\prime}$, then

$$
\bar{W}\left(p_{i}, \alpha\right)<\bar{W}(q, 2 \alpha),
$$

therefore, we have

$$
\phi\left\{\bar{W}\left(p_{i}, \alpha\right)\right\} \leqq \phi\{\bar{W}(q, 2 \alpha)\} .
$$

By (2), (3) and (4) we have

$$
\phi\left(W_{i}^{\prime}\right)<\frac{1}{\gamma^{m-1}} \phi\{\bar{W}(q, 2 \alpha)\} .
$$

But this relation holds for any value of $i$, therefore we have

but since

$$
\sum_{i=1}^{k} \phi\left(W_{i}^{\prime}\right)<\frac{k}{\gamma^{m-1}} \phi\{\bar{W}(q, 2 \alpha)\},
$$

and

$$
\phi\left(Q_{\alpha} W^{\prime}\right) \leqq \sum_{i=1}^{k} \phi\left(W_{i}^{\prime}\right)
$$

$$
k \leqq 2^{m+1}
$$

we have

$$
\phi\left(Q_{\alpha} W^{\prime}\right) \leqq 4\left(\begin{array}{l}
2 \\
\gamma
\end{array}\right)^{m-1} \phi\{\bar{W}(q, 2 \alpha)\}
$$

Since $\frac{2}{\gamma}<1$, and the above relation holds for any value of $m$ however great, it must be that

$$
\phi\left(Q_{\alpha} W^{\prime}\right)=0 \text {. }
$$

Since this relation holds for every mesh $W^{\prime}$, we have

$$
\phi\left(Q_{\alpha}\right)=0 \text {. }
$$

Now let $\left\{\alpha_{n}\right\}$ be a sequence of positive numbers converging to zero, then

$$
Q=Q_{\alpha_{1}} \dot{+} Q_{\alpha_{2}} \dot{+} \ldots \ldots \dot{+} Q_{\alpha_{n}} \dot{+} \ldots \ldots
$$

But by (5),

$$
\phi\left(Q_{\alpha_{n}}\right)=0, \quad(n=1,2, \ldots)
$$

therefore, we have the result

$$
\phi(Q)=0 .
$$


3. Let $\Phi(E)$ be a completely additive function of normal sets defined in $\mathfrak{F}$; then we give the definition of the general derivatives as follows: If there exists a $\phi$-regular monotone sequence $\left\{M_{i}\right\}$ converging to a point $a$, so that

$$
\lim _{i \rightarrow \infty} \frac{\Phi\left(M_{i}\right)}{\phi\left(M_{i}\right)}
$$

exists, then this limit is said to be a general derivative of $\Phi(E)$, at $a$, with respect to $\phi(E)$, and denoted by $D_{\dot{\phi}} \Phi$. From the preceding section, except the set of essential poles, i.e. almost everywhere $(\phi)$ the general derivatives exist and their values depend on the $\phi$-regular monotone sequence of point sets.

Now I will proceed to prove the fundamental theorem :

$\Phi(E)$ has a unique finite general derivative $D_{\phi} \Phi$ almost everywhere (ф) in $A$, and

$$
\Phi(A)=\Phi\left(A^{\times \times}\right)^{(1)}+\int_{A} D_{\phi} \Phi d \phi,
$$

for any set $A$ of $\mathfrak{F}$.

To prove this theorem for Euclidian space, there are two methods,

(i) using the net derivative,

(ii) using the extension of Vitali's theorem.

But for either method, Daniell's $(s, q)$ complex of nets is an useful aid as I will explain in the next section.

\section{Proof of the Fundamental Theorem.}

4. Consider a net of which the $x_{i}$-coordinates of the knots are of the form

$$
t\left(\alpha_{i}+\frac{p_{i}}{2^{n}}\right)
$$

where $p_{i}$ is a positive or negative integer or zero, $\alpha_{i}$ is zero or a proper rational fraction of the form

$$
\alpha_{i}=\frac{r_{i}}{q} \quad\left(r_{i}=0,1,2, \ldots, q-1\right)
$$

(1) $A \times \times$ is the singular-part of $A$ for $\Phi(E)$ on the base $\phi(E)$; therefore, when $\Phi(E)$ is absolutely continuous with respect to $\phi(E), A^{\times \times}$is empty. 
with $q$ an odd positive integer, and where $t$ is a positive number of the form

$$
t=2^{\frac{j}{s}} \quad(j=0,1,2, \ldots, s-1)
$$

with $s$ a positive integer.

By keeping $t, \alpha_{i}$, and $n$ fixed, and varying $p_{i}$ we have a net of order $n$; the whole set of nets for $n=1,2, \ldots$ is called a net system; if, in addition, we vary $r_{i}$ by taking a fixed value $q$, we have $q^{k}$ net systems which are called associated net systems. Furthermore, by taking a fixed value $s$ and varying $j$, we obtain $s q^{k}$ associated net systems, the whole set of which is called by Daniell an $(s, q)$ complex of nets.

Concerning this $(s, q)$ complex of nets, we have the following important lemma which I owe to Daniell. ${ }^{(1)}$

Corresponding to a positive number $\lambda$ which is greater than 1 , there exists an $(s, q)$ complex of nets, so that for any point a, there is a mesh $W^{\prime}$ of order $n$ belonging to one of the $s q^{k}$ associated net systems, which satisfies the following relation

$$
\bar{W}(a, \rho)<W^{\prime}<\bar{W}(a, \lambda \rho),
$$

where $\rho$ is a sufficiently small positive number.

Since $1+\lambda$ is greater than 2 , there exists an odd integer $q$ so that

$$
(1+\lambda) \frac{q-1}{q}>2 \text {. }
$$

If we take a sufficiently great integer $s$, then it may be that

$$
\frac{1}{s}<\log _{2}\left\{(1+\lambda) \frac{q-1}{q}\right\}-1 \text {. }
$$

Thus I determined $q$ and $s$, i.e. the $(s, q)$ complex of nets which corresponds to $\lambda$. Now I will show that there is a mesh $W^{\prime}$ which satisfies (1).

Given any sufficiently small positive number $\rho$, it is possible to choose positive integers $n$ and $j$ so that

$$
\log _{2} \frac{q}{q-1}+\log _{2} \rho<\frac{j}{s}-n<\log _{2}(1+\lambda)+\log _{2} \rho-1
$$

(1) Loc. cit., 191. 
From this we have

$$
\begin{aligned}
& \frac{q-1}{q} \frac{t}{2^{n}}>\rho, \\
& \frac{t}{2^{n}}<(1+\lambda) \frac{\rho}{2},
\end{aligned}
$$

where $t=2^{\frac{j}{s}}$.

From (2), there cannot be more than $q-1$ consecutive numbers of the form

$$
t\left(\frac{r_{i}}{q}+\frac{p_{i}}{2^{n}}\right)
$$

in $\left(a_{i}-\frac{\rho}{2}, a_{i}+\frac{\rho}{2}\right)$, where $a_{i}$ is the $x_{i}$-coordinate of the point $a$. Therefore, in the $x_{i}$-coordinate we can pick out a mesh interval $\left(\alpha_{i}, \beta_{i}\right)$ belonging to a net $N$ of order $n$, covering $\left(a_{i}-\frac{\rho}{2}, a_{i}+\frac{\rho}{2}\right)$.

But by (3) there must be a knot of the net $N$ in $\left(a_{i}-\lambda \frac{\rho}{2}, a_{i}+\frac{\rho}{2}\right)$. This cannot be between $a_{i}-\frac{\rho}{2}$ and $a_{i}+\frac{\rho}{2}$, so that it must lie between $a_{i}-\lambda \frac{\rho}{2}$ and $a_{i}-\frac{\rho}{2}$. Hence $\alpha_{i}$ must be between these values. Similarly $\beta_{i}$ must be between $a_{i}+\frac{\rho}{2}$ and $a_{i}+\lambda \frac{\rho}{2}$.

Let $W^{\prime}$ be the mesh which has the interval $\left(\alpha_{i}, \beta_{i}\right)$ for its side in $x_{i}$-coordinate. Then $W^{\prime}$ is a mesh of order $n$ which belongs to one of the $s q^{k}$ associated net systems of the $(s, q)$ complex of nets and satisfies the relations

$$
\bar{W}(p, \rho)<W^{\prime}<\bar{W}(p, \lambda \rho) .
$$

5. To prove the fundamental theorem of section 3 using the net derivative, it is sufficient to prove the following theorem; since all the other investigations of the previous paper ${ }^{(1)}$ can be used here as they stand.

If $\Phi(E)$ be non-negative, and if the net derivative $D_{\phi}^{(n)} \Phi$ is zero for all net systems of all $(s, q)$ complexes of nets with $s=1,2,3, \ldots, q=1$, $3,5, \ldots$, then the general derivative $D_{\phi} \Phi$ is also zero almost everywhere $(\phi)$.

(1) Loc. cit., 16-20. 
To prove this, let $M$ be a set which belongs to the $\phi$-regular monotone sequence of point sets converging to a point $a$, so that

$$
\begin{aligned}
& M \leqq \bar{W}(a, \rho), \\
& \frac{\phi(M)}{\phi\{\bar{W}(a, \rho)\}} \geq \vartheta(a), \\
& \frac{\phi\{\bar{W}(a, \rho)\}}{\phi\{\bar{W}(a, \lambda \rho)\}} \geq \kappa(a, \lambda),
\end{aligned}
$$

then by the lemma of section 4 , there exists a mesh $W^{\prime}$ belonging to one of the associated nets of the $(s, q)$ complex of nets which corresponds to $\lambda$, and

$$
\bar{W}(a, \rho)<W^{\prime}<\bar{W}(a, \lambda \rho) .
$$

Therefore, we have

$$
\begin{aligned}
\frac{\phi(M)}{\phi(M)} \leqq \frac{1}{\vartheta(a)} \frac{\phi\{\bar{W}(a, \rho)\}}{\phi\{\bar{W}(a, \rho)\}} & \leqq \frac{1}{\vartheta(a)} \frac{\phi\{\bar{W}(a, \lambda \rho)\}}{\phi\{\bar{W}(a, \rho)\}} \frac{\Phi\left(W^{\prime}\right)}{\phi\left(W^{\prime}\right)} \\
& \leqq \frac{1}{\vartheta(a)_{\kappa}(a, \lambda)} \frac{\phi\left(W^{\prime}\right)}{\phi\left(W^{\prime}\right)}
\end{aligned}
$$

Since $\lim \frac{\phi\left(W^{\prime}\right)}{\phi\left(W^{\prime}\right)}$ is a net derivative at $a$ and zero by hypothesis, we conclude that the general derivative $\lim \frac{\phi(M)}{\phi(M)}$ at $a$ is zero.

6. For the proof of the fundamental theorem of section 3 Daniell(1) discarded the investigation based on an extension of Vitali's theorem on the ground that for that purpose it would have been necessary to consider only the case where $\lambda \geqq 3$. But here I will give a proof of this theorem for $\lambda>1$ using his $(s, q)$ complex of nets.

Now the extension of Vitali's theorem is stated as follows:

Let $\phi(E)$ be completely additive, non-negative, function of normal sets, and $A$ be a $\phi$-normal set where $\phi(A) \neq 0$. If at any point a of $A$ there is a $\phi$-monotone sequence of neighbourhoods, then from these we can select an enumerable, or a finite, set of neighbourhoods

$$
\bar{W}\left(a_{1}, \rho_{1}\right), \bar{W}\left(a_{2}, \rho_{2}\right), \ldots \ldots, \bar{W}\left(a_{n}, \rho_{n}\right), \ldots \ldots,
$$

(1) Lo:. cit., 198. 
so that

1. no two of these have a point in common, and

2. if we denote the sum of (1) by $S$, then

$$
\phi(A-A S)=0 \quad \text { and } \quad \phi(S-A S)<\varepsilon
$$

for any given positive number $\epsilon$.

(i) Let $O$ be a an open set which contains $A$, and assume that there exists a fixed number $\theta$ so that we can find a sequence of neighbourhoods

$$
\bar{W}\left(a_{1}, \rho_{1}\right), \quad \bar{W}\left(a_{2}, \rho_{2}\right), \quad \ldots \ldots \ldots
$$

which are contained in $O$, and no two of which have a point in common, and

$$
\phi\left\{\bar{W}\left(\alpha_{1}, \rho_{1}\right)+\bar{W}\left(a_{2}, \rho_{2}\right)+\ldots .\right\}>\theta \phi(A) .
$$

Then from this assumption, as was done by Schlesinger and Plessner ${ }^{(1)}$, we can prove that Vitali's theorem holds.

For, let $\nu_{1}$ be a sufficiently great number, then from (1) we have

$$
\phi\left\{\bar{W}\left(a_{1}, \rho_{1}\right)\right\}+\phi\left\{\bar{W}\left(a_{2}, \rho_{2}\right)\right\}+\ldots .+\phi\left\{\bar{W}\left(a_{\nu_{1}}, \rho_{\nu_{1}}\right)\right\}>\theta \phi(A) .
$$

Let $S_{1}$ be the sum of

$$
\bar{W}\left(a_{1}, \rho_{1}\right), \quad \bar{W}\left(a_{2}, \rho_{2}\right), \ldots \ldots, \quad \bar{W}\left(a_{\nu_{1}}, \rho_{\nu_{1}}\right),
$$

then $S_{1}$ being closed, $O-S_{1}$ is open, and

$$
A-A S_{1} \cong O-S_{1} \text {. }
$$

Using $A-A S_{1}$ and $O-S_{1}$ instead of $A$ and $O$, by the assumption, there exists a sequence of neighbourhoods which are contained in $O-S_{1}$, and no two of which have a point in common, so that the $\phi$ value of the sum of these neighbourhoods is greater than $\theta \phi\left(A-A S_{1}\right)$. Let, as above, $S_{2}$ be the sum of first $\nu_{2}$ neighbourhoods of this sequence, then

and

$$
S_{1} S_{2}=0 \text {, }
$$$$
\phi\left(S_{2}\right)>\theta \phi\left(A-A S_{1}\right) .
$$

(1) Lebesguesche Integale und Fouriersche Reihen, (1926), 117. 
Repeating this process $p$ times, we have

$$
\phi\left(S_{p}\right)>\theta \phi\left(A-A S_{1}-A S_{2}-\ldots . .-A S_{p-1}\right) .
$$

Let $p$ be indefinitely great and put

$$
S=S_{1}+S_{2}+\ldots \ldots+S_{p}+\ldots \ldots
$$

then $S$ being contained in $O, \phi(S)$ has a finite value. Since the series

$$
\phi\left(S_{1}\right)+\phi\left(S_{2}\right)+\ldots .+\phi\left(S_{p}\right)+\ldots .
$$

converges to $\phi(S)$, it must be that

$$
\lim _{p \rightarrow \infty} \phi\left(S_{p}\right)=0 .
$$

Therefore, by (2) we have

$$
\phi(A-A S)=0 .
$$

$\phi(A)$ being normal $(\phi)$, it is possible to choose the above set $O$ so that

$$
\phi(O)<\phi(A)+\varepsilon,
$$

where $\varepsilon$ is the given positive number. Since

we have

$$
\phi(S) \leqq \phi(O),
$$

$$
\phi(S)<\phi(A)+\varepsilon .
$$

But from (3)

$$
\phi(A)=\phi(A S),
$$

therefore, we have

$$
\phi(S-A S)<\varepsilon .
$$

Thus from the assumption we can prove Vitali's theorem.

(ii) Now, using the $(s, q)$ complex of nets, I will show that the assumption of (i) is true. For that purpose, first consider the case where at any point $a$ of $A$ there exists a $\phi$-monotone sequence of neighbourhoods $\left\{\bar{W}\left(a, \rho_{i}^{(a)}\right)\right\}$ which satisfies

$$
\frac{\phi\left\{\bar{W}\left(a, \rho_{i}^{(a)}\right)\right\}}{\phi\left\{\bar{W}\left(a, \lambda \rho_{i}^{(\alpha)}\right)\right\}} \geqq \kappa(A, \lambda),
$$


where $\lambda$ and $\kappa(A, \lambda)$ are constant numbers independent of the points of $A$. Then to this $\lambda$, by the lemma of section 4 , there corresponds an $(s, q)$ complex of nets which has $s q^{k}$ associated net systems.

For any point $a$ of $A$, from the sequence of neighbourhoods pick out one, $\bar{W}(a, \rho)$, which is contained in $O$ of (i), $\rho$ being sufficiently small. Then by the lemma, there exists a mesh $W^{\prime}$ of order $n$ belonging to one of the $s q^{k}$ associated net systems, and

$$
\bar{W}(a, \rho)<W^{\prime}(a)<\bar{W}(a, \lambda \rho) .
$$

Take this mesh $W^{\prime}(a)$ for all points $a$ of $A$, and put in one class all the meshes which belong to the same associated net systems, then we have $s q^{k}$ classes of meshes. But since the set of all these meshes covers the set $A$, one of the $s q^{k}$ classes of the meshes covers a part $B$ of $A$ where

$$
\phi(B) \geqq \frac{\phi(A)}{s q^{k}} .
$$

From the meshes belonging to this class, remove those which are included in other meshes, then we have a sequence of meshes

$$
W^{\prime}\left(a_{1}\right), \quad W^{\prime}\left(a_{2}\right), \quad \ldots \ldots \ldots
$$

no two of which have a point in common, and the sum of (6) covers $B$. That is,

$$
\sum_{i} \phi\left\{W^{\prime}\left(a_{i}\right)\right\} \geqq \frac{\phi(A)}{s q^{k}} .
$$

To each $W^{\prime}\left(a_{i}\right)$ take the corresponding neighbourhood $\bar{W}\left(a_{i}, \rho_{i}\right)$ which satisfies the relation (5), then no two of the neighbourhoods

$$
\bar{W}\left(a_{1}, \rho_{1}\right), \bar{W}\left(a_{2}, \rho_{2}\right), \ldots \ldots, \bar{W}\left(a_{i}, \rho_{i}\right), \ldots \ldots
$$

have a point in common, and

$$
\sum_{i} \phi\left\{\bar{W}\left(a_{i}, \lambda \rho_{i}\right)\right\} \geqq \frac{\phi(A)}{s q^{k}} .
$$

But by (4), we have

$$
\sum_{i} \phi\left\{\bar{W}\left(a_{i}, \rho_{i}\right)\right\} \geqq \frac{\kappa(A, \lambda)}{s q^{k}} \phi(A) .
$$


Let $\theta$ be a number smaller than $\frac{\kappa(A, \lambda)}{s q^{k}}$, then we have

$$
\phi\left\{\bar{W}\left(a_{1}, \rho_{1}\right)+\bar{W}\left(a_{2}, \rho_{2}\right)+\ldots .\right\}>\theta \phi(A) ;
$$

therefore, in this case the assumption of ( $i$ ) is true.

(iii) If at every point $a$ of $A$, there exists a $\phi$-monotone sequence of neighbourhoods with respect to the same value of $\lambda$, the lower bound of $\kappa(a, \lambda)$ being zero, then denote, as Schlesinger and Plessner ${ }^{(1)} \mathrm{did}$, the set of points where $\kappa(a, \lambda) \geqq \frac{1}{\nu}$ by $A_{\nu}$, where $\nu$ is a natural number. Then

$$
A_{1} \leqq A_{2} \subseteq \ldots . \subseteq A_{\nu} \leqq \ldots
$$

and

$$
A=\operatorname{Lim}_{\nu \rightarrow \infty} A_{\nu} \text {. }
$$

Therefore, since

$$
\phi(A)=\lim _{\nu \rightarrow \infty} \phi\left(A_{\nu}\right),
$$

for a sufficiently great number $\nu$

$$
\phi\left(A_{\nu}\right)>\frac{1}{2} \phi(A) .
$$

But at at every point $a$ of $A_{\nu}, \kappa(a, \nu) \geqq \frac{1}{\nu}$; therefore, by (ii) there exists a sequence of neighbourhoods $\left\{\bar{W}\left(a_{i}, \rho_{i}\right)\right\}$ which are contained in $O$, and no two of which have a point in common, and

$$
\phi\left\{\bar{W}\left(a_{1}, \rho_{1}\right)+\bar{W}\left(a_{2}, \rho_{2}\right)+\ldots .\right\} ? \theta^{\prime} \phi\left(A_{\nu}\right) .
$$

Combining with (7) we get

$$
\phi\left\{\bar{W}\left(a_{1}, \rho_{1}\right)+\bar{W}\left(a_{2}, \rho_{2}\right)+\ldots .\right\} ? \frac{\theta^{\prime}}{2} \phi(A) ;
$$

therefore, by putting $\theta=\frac{\theta^{\prime}}{2}$ we see that the assumption of (i) is true in this case.

(iv) Lastly we consider the most general case where the values of $\lambda$ depend on the point $a$ and the lower bound of $\lambda$ is 1 . Let $A^{(\nu)}$ be the set of points $a$ of $A$ where $\lambda \geq 1+\frac{1}{\nu}$. Then

$$
A^{(1)} \leqq A^{(2)} \leqq \ldots . \subseteq A^{(2)} \leqq \ldots .
$$

(1) Loc. cit., 118. 
and

$$
A=\operatorname{Lim}_{\nu \rightarrow \infty} A^{(\nu)}
$$

Therefore, since $\phi(A)=\lim _{\nu \rightarrow \infty} \phi\left(A^{(\nu)}\right)$,

for a sufficiently great number $\nu$

$$
\phi\left(A^{(2)}\right)>\frac{1}{2} \phi(A) .
$$

But at every point $a$ of $A^{(v)}$, the sequences of neighbourhoods are $\phi$-monotone with respect to $\lambda=1+\frac{1}{\nu}$; then by (iii) there exists a sequence of neighbourhoods $\bar{W}\left(a_{i}, \rho_{i}\right)$ which are contained in $O$, and no two of which have a point in common, and

$$
\phi\left\{\bar{W}\left(a_{1}, \rho_{1}\right)+\bar{W}\left(a_{2}, \rho_{2}\right)+\ldots .\right\} \geqq \theta^{\prime \prime} \phi(A) .
$$

Combining with (8), we have

$$
\phi\left\{\bar{W}\left(a_{1}, \rho_{1}\right)+\bar{W}\left(a_{2}, \rho_{2}\right)+\ldots \ldots\right\} \geqq \frac{\theta^{\prime \prime}}{2} \phi(A),
$$

therefore, by putting $\theta=\frac{\theta^{\prime \prime}}{2}$, we see that the assumption of (i) is true in this most general case.

Thus we have proved the extension of Vitali's theorem. After this theorem has been proved, the demonstration of the fundamental theorem is almost the same as that of my previous paper ${ }^{(1)}$, except for the considerations concerning the set of poles $A_{p}$.

7. Let $\Phi(E)$ be an integral with respect to $\phi(E)$, i. e.,

$$
\Phi(E)=\int_{E} f(p) d \phi,
$$

then $\Phi(E)$ is absolutely continuous with respect to $\phi(E)$. Therefore, combining this with the above proved fundamental theorem, we have the following theorem :

The necessary and sufficient condition that a function of normal sets $\Phi(E)$ may be an integral with respect to another function of normal sets $\phi(E)$ is that $\Phi(E)$ is absolutely continuous with respect to $\phi(E)$.

(1) Loc. cit., 10-14. 
Then, what is the condition necessary and sufficient in order that a bounded $\phi$-measurable function $f(p)$ may be a unique general derivative of a set function $\Phi(E)$ with respect to $\phi(E)$ ?

Concerning the derivative with respect to the Lebesgue measure, Denjoy ${ }^{(1)}$ has proved that the approximate continuity is a sufficient condition, but, in giving an example, he said that this condition is not necessary. But the derivative he used is a special one and not the general derivative. In respect of this general derivative I will prove, in the following section, that the approximate continuity is not only a sufficient but also a necessary condition. For this purpose I will first consider the approximate continuity of point functions in detail.

\section{Definition of the Approximate Continuity.}

8. Let $\phi(E)$ be a non-negative function of normal sets, and let $\mathfrak{F}$ denote the closed family which contains all the $\phi$-normal set. Let $A$ be a $\phi$-normal set, and consider a completely additive set function

$$
\psi(E)=\phi(E A)=\int_{E} h(p) d \phi
$$

defined in $\mathfrak{F}$, where $h(p)$ is the characteristic function of $A$. Then, $\psi(E)$ is also a function of normal sets. Therefore, by the fundamental theorem $\psi(E)$ has a unique general derivative which is equal to 1 almost everywhere $(\phi)$ in $A$. I call this derivative the $\phi$-density of the set $A$. This is the extension of the metric density of the set with respect to the Lebesgue measure.

9. Let $f(p)$ be a $\phi$-measurable point function which is defined in a $\phi$-normal set $P$. Then $f(p)$ is said to be approximately continuous with respect to $\phi(E)$ at a point $p$ of $P$, when, corresponding to each arbitrarily chosen number $\varepsilon$, the set of points $q$ for which

$$
|f(q)-f(p)|<\varepsilon
$$

has the $\phi$-density unity at the point $p$.

Concerning the approximate continuity we have the following theorem :

The necessary and sufficient condition that the function $f(p)$ should be approximately continuous with respect to $\phi(E)$ at the point $p$, is that

(1) M. A. Denjoy, Bull. de la Soc. Math. de France, 43 (1915), 172 and 178. 
it should be continuous, at $p$, relatively to a point set $Q$ which has the $\phi$-density unity at the point $p$.

We can prove this theorem modifying Denjoy's(1) method as follows :

The condition in the theorem is sufficient; for there exists a number $\rho$, such that in the interval $W(p, \rho)$, the condition

$$
|f(q)-f(p)|<\epsilon
$$

is satisfied for every point $q$ that belongs to $Q$. This part of $Q$ has the same $\phi$-density at $p$ as $Q$ itself.

To show that the condition is necessary, let it be assumed that the set $Q(p, \varepsilon)$ of points at which $|f(q)-f(p)|<\epsilon$ has the $\phi$-density 1 at the point $p$, whatever value $\varepsilon$ may have.

Let $\left\{\varepsilon_{n}\right\}$ be a sequence of decreasing values of $\epsilon$ that converges to zero, and $\left\{M_{i}\right\}$ be any $\phi$-regular monotone sequence of point sets convering to the point $p$. For each $\epsilon_{n}$ a number $i$ can be so determined that the $\phi$-value of the part of $Q\left(p, \epsilon_{n}\right)$ in the set $M_{i}$ is greater than $\phi\left(M_{i}\right)\left(1-\varepsilon_{n}\right)$; then $i$ becomes infinite with $n$. Take a sufficiently small number $\rho_{n}$ so that the $\phi$-value of the part of $Q\left(p, \epsilon_{n}\right)$ in the set $M_{i} W\left(p, \rho_{n}\right)$ is less than $\varepsilon_{n} \phi\left(M_{i}\right)$.

Let $Q_{n}$ be the part of $Q\left(p, \varepsilon_{n}\right)$ that is in $M_{i}-M_{i} W\left(p, \rho_{n}\right)$. If $Q$ be the set $Q_{1}+Q_{2}+\ldots+Q_{n}+\ldots$, then the set $Q$ has the $\phi$-density unity at the point $p$, since

$$
\phi\left(Q M_{i}\right) \geqq \phi\left(Q_{n}\right)>\phi\left(M_{i}\right)\left(1-\epsilon_{n}\right)-\varepsilon_{n} \phi\left(M_{i}\right)=\phi\left(M_{i}\right)\left(1-2 \epsilon_{n}\right) .
$$

Let $p_{1}, p_{2}, \ldots, p_{m}, \ldots$ be any sequence of points converging to $p$, and such that all of them are points of $Q$. Each one of them $p_{m}$ belongs to one of the sets $Q_{1}, Q_{2}, \ldots, Q_{n}, \ldots$, say to $Q_{n}$. It is clear that $n$ becomes infinite as $m$ does so. We have then

$$
\left|f\left(p_{m}\right)-f(p)\right|<\epsilon_{n} ;
$$

and therefore, the sequence $\left\{f\left(p_{m}\right)\right\}$ converges to $f(p)$. Hence $f(p)$ is continuous relatively to $Q$, which has the $\phi$-density unity at $p$.

\section{Approximate Continuity of the General Derivative.}

10. Let $\phi(E)$ be a non-negative function normal sets, and $f(p)$ be a bounded $\phi$-measurable point function. Consider the set function defined in $\mathfrak{F}$,

(1) Loc. cit., 166-168; cf. E.W. Hobson, The theory of functions of a real variable, I, third ed. (1927), 313. 


$$
\Phi(E)=\int_{E} f(p) d \phi
$$

By the fundamental theorem, almost everywhere $(\phi) \quad \Phi(E)$ has a unique general derivative $D_{\phi} \Phi$ which is equivalent $(\phi)$ to $f(p)$. But this theorem tells us nothing as to the points at which the general derivative $D_{\phi} \Phi$ is equal to $f(p)$. For this contingency the following theorem :

The necessary and sufficient condition that $\phi(E)$ may have a unique general derivative $D_{\phi} \Phi$ which is equal to $f(p)$ at a point $p_{0}$, is that $f(p)$ is approximately continuous at $p_{0}$ with respect to $\phi(E)$.

This condition is sufficient ${ }^{(1)}$. For, let $\epsilon$ be any given positive number, then the set $A$ of points $p$ where

$$
\left|f(p)-f\left(p_{0}\right)\right|<\epsilon
$$

has the $\phi$-density unity at $p_{0}$. That is, let $\left\{M_{i}\right\}$ be any $\phi$-regular monotone sequence of point sets converging to $p_{0}$, then

$$
\lim _{i \rightarrow \infty} \frac{\phi\left(M_{i} A\right)}{\phi\left(M_{i}\right)}=1
$$

Therefore, corresponding to any arbitrarily chosen positive number $\eta$, a natural number $i_{0}$ exists, so that

$$
\phi\left(M_{i} A\right) \geqq(1-\eta) \phi\left(M_{i}\right)
$$

for all values of $i$ which are greater than $i_{0}$.

Now

$$
\Phi(E)=\int_{E} f(p) d \phi=f\left(p_{0}\right) \Phi(E)+\int_{E}\left\{f(p)-f\left(p_{0}\right)\right\} d \phi
$$

therefore, if we denote

$$
\int_{E}\left\{f(p)-f\left(p_{0}\right)\right\} d \phi
$$

by $\Phi_{1}(E)$ then

$$
\frac{\phi\left(M_{i}\right)}{\phi\left(M_{i}\right)}=f\left(p_{c}\right)+\frac{\Phi_{1}\left(M_{i}\right)}{\phi\left(M_{i}\right)}
$$

(1) This proof of the sufficient condition is due to Denjoy ; loc. cit., 172. 
But we have

$$
\left|\frac{\Phi_{1}\left(M_{i}\right)}{\phi\left(M_{i}\right)}\right| \leqq\left|\frac{\Phi_{1}\left(M_{i} A\right)}{\phi\left(M_{i}\right)}\right|+\left|\frac{\Phi_{1}\left(M_{i}-M_{i} A\right)}{\phi\left(M_{i}\right)}\right| .
$$

Since at the points of $A$ the relation (1) holds,

$$
\left|\Phi_{1}\left(M_{i} A\right)\right|<\epsilon \phi\left(M_{i} A\right) \leqq \epsilon \phi\left(M_{i}\right) .
$$

And $f(p)$ being bounded, there exists a definite positive number $\alpha$ so that $|f(p)|<\alpha$; then since $\left|f(p)-f\left(p_{0}\right)\right|<2 \alpha$,

$$
\left|\Phi_{1}\left(M_{i}-M_{i} A\right)\right|<2 \alpha \phi\left(M_{i}-M_{i} A\right) \text {. }
$$

But by (2) we have

$$
\Phi\left(M_{i}-M_{i} A\right)=\phi\left(M_{i}\right)-\phi\left(M_{i} A\right) \leqq \eta \phi\left(M_{i}\right)
$$

for $i>i_{0}$.

Combining (5) and (6) with (4), we have

$$
\left|\frac{\phi_{1}\left(M_{i}\right)}{\phi\left(M_{i}\right)}\right| \leqq \epsilon+2 \alpha \eta
$$

for $i>i_{0} ; \varepsilon$ and $\eta$ being any positive number however small, we have

$$
\lim _{i \rightarrow \infty} \frac{\Phi_{1}\left(M_{i}\right)}{\phi\left(M_{i}\right)}=0
$$

Then by (3) we have

$$
\lim _{i \rightarrow \infty} \frac{\Phi\left(M_{i}\right)}{\phi\left(M_{i}\right)}=f\left(p_{0}\right) .
$$

But since $\left\{M_{i}\right\}$ is an arbitrarily chosen $\phi$-regular monotone sequence converging to $p_{0},(7)$ shows that $\Phi(E)$ has a unique general derivative $D_{\phi} \Phi$ which is equal to $f\left(p_{0}\right)$.

Next, the approximate continuity of $f(p)$ is a necessary condition. Since, if $f(p)$ is not approximately continuous at $p_{0}$, then there exists a positive number $\sigma$ so that, denoting the set of points $p$. at which

$$
f(p)-f\left(p_{0}\right)>\sigma
$$

by $B$, and the set of points $p$ at which

$$
f\left(p_{0}\right)-f(p)>\sigma
$$


by $C$, at least one of these sets, $B$ or $C$, cannot have the $\phi$-density zero at $p_{0}$. Now assume that the $\phi$-density of $B$ is not zero. Let $\eta$ be a sufficiently small number, then there exists a $\phi$-regular monotone sequence $\left\{M_{i}\right\}$ converging to $p_{0}$ and a natural number $i_{0}$ so that

$$
\frac{\phi\left(M_{i} B\right)}{\phi\left(M_{i}\right)}>\eta
$$

for $i>i_{0}$.

Since $\left\{M_{i}\right\}$ is $\phi$-regular monotone, the following conditions hold :

$$
\left.\begin{array}{l}
M_{i} \subseteq \bar{W}\left(p_{0}, \rho_{i}\right), \\
\frac{\phi\left(M_{i}\right)}{\phi\left\{\bar{W}\left(p_{c}, \rho_{i}\right)\right\}} \geqq \vartheta\left(p_{0}\right) \\
\frac{\phi\left\{\bar{W}\left(p_{0}, \rho_{i}\right)\right\}}{\phi\left\{\bar{W}\left(p_{0}, \lambda \rho_{i}\right)\right\}} \geqq \kappa\left(p_{0}, \lambda\right)
\end{array}\right\}
$$

Let $N_{i}=M_{i} B$, then the sequence $\left\{N_{i}\right\}$ is also $\phi$-regular monotone for $i>i_{0}$, since by (8) and (9) we have

$$
\begin{aligned}
& N_{i} \subseteq \bar{W}\left(p_{0}, \rho_{i}\right), \\
& \frac{\phi\left(N_{i}\right)}{\phi\left\{W\left(p_{0}, \rho_{i}\right)\right\}} \geqq \eta \vartheta\left(p_{0}\right), \\
& \frac{\phi\left\{\bar{W}\left(p_{0}, \rho_{i}\right)\right\}}{\phi\left\{\bar{W}\left(p_{0}, \lambda \rho_{i}\right)\right\}} \geqq \kappa\left(\rho_{0}, \lambda\right) .
\end{aligned}
$$

Now

$$
\frac{\Phi\left(N_{i}\right)}{\phi\left(N_{i}\right)}=f\left(p_{0}\right)+\frac{\Phi_{1}\left(N_{i}\right)}{\phi\left(N_{i}\right)}
$$

But since at any point $p$ of $N_{i}, f(p)-f\left(p_{0}\right)>\sigma$, and $\phi(E)$ is non-negative, we have

$$
\Phi_{1}\left(N_{i}\right)>\sigma \phi\left(N_{i}\right) .
$$

Therefore, $\frac{\phi\left(N_{i}\right)}{\phi\left(N_{i}\right)}$ does not converge to $f\left(p_{0}\right)$, that is, $\phi(E)$ cannot have a unique general derivative which is equal to $f\left(p_{0}\right)$.

The theorem aboved proved is also stated as follows :

The necessary and sufficient condition that a bounded $\phi$-measurable point function $f(p)$ may be a unique general derivative of a set function 
with respect to $\phi(E)$, is that $f(p)$ is approximately continuous with respect to $\phi(E)$ at all points.

Combining this with the fundamental theorem, we can say that all the bounded $\phi$-measurable point functions are approximately continuous with respect to $\phi(E)$ almost everywhere $(\phi)$.

But the boundedness of the point function can be removed as was done by Hobson ${ }^{(1)}$, and we have the following theorem :

All the $\phi$-measurable point functions, finite almost everywhere $(\phi)$, are approximately continuous with respect to $\phi(E)$ almost everywhere $(\phi)$.

i1. As in section 7 cited, Denjoy gave the following example :

$$
\begin{aligned}
& F(x)=x^{2} \sin \frac{1}{x}, \\
& \left\{\begin{array}{l}
f(x)=-\cos \frac{1}{x}+2 x \sin \frac{1}{x}, \quad x \neq 0 \\
f(0)=0,
\end{array}\right.
\end{aligned}
$$

and said that although $f(x)$ is bounded and not approximately continuous at $x=0$, it is the derivative of $F(x)$ at $x=0$.

But the derivative he considered is a special one, i.e. the derivative of the ordinary differential calculus, and this example does not conflict with the theorem of section 10 . Since $F(x)$ is continuous and of bounded variation, we can turn this into a function of normai sets $\Phi(E)^{(2)}$. In what follows, I will show that the general derivatives of $\phi(E)$ with respect to the Lebesgue measure $m(E)$ are not unique at $x=0$.

Denote the linear interval

$$
\frac{1}{\left(2 n+\frac{1}{2}\right) \pi} \leqq x \leqq \frac{1}{2 n \pi}
$$

by $I_{n}$, and consider the point set

$$
M_{i}=I_{i}+I_{i+1}+\ldots \ldots+I_{2 i-1} \text {. }
$$

Then $\left\{M_{i}\right\}$ is a $m$-regular sequence of point sets which converges to $x=0$. For, since

(1) The theory of functions of a real variable, II, second ed. (1926), 257.

(2) Cf. de la Vallée Poussin, Intégrales de Lebesgue, (1916), 99. 


$$
\begin{aligned}
& m\left(I_{n}\right)=\frac{1}{2 n \pi}-\frac{1}{\left(2 n+\frac{1}{2}\right) \pi}=\frac{1}{8 n\left(n+\frac{1}{4}\right) \pi}, \\
& m\left(M_{i}\right)=\frac{1}{8 \pi} \sum_{n=i}^{2 i-1} \frac{1}{n\left(n+\frac{1}{4}\right)}>\frac{1}{8 \pi} \sum_{n=i}^{2 i-1} \frac{1}{n(n+1)}=\frac{1}{16 i \pi} .
\end{aligned}
$$

But $M_{i}$ is contained in the interval $\bar{W}\left(0, \frac{1}{i \pi}\right)$ whose measure is $\frac{1}{i \pi}$, therefore

$$
\frac{m\left(M_{i}\right)}{m\left\{\bar{W}\left(0, \frac{1}{i \pi}\right)\right\}}>\frac{1}{16}
$$

Now

$$
\Phi\left(I_{n}\right)=F\left(\frac{1}{2 n \pi}\right)-F\left(\frac{1}{\left(2 n+\frac{1}{2}\right) \pi}\right)=-\frac{1}{\left(2 n+\frac{1}{2}\right)^{2} \pi^{2}}
$$

therefore,

$$
\frac{\Phi\left(M_{i}\right)}{m\left(M_{i}\right)}=-\frac{\sum_{n=i}^{2 i-1} \frac{1}{\left(2 n+\frac{1}{2}\right)^{2} \pi^{2}}}{\sum_{n=i}^{2 i-1} \frac{1}{8 n\left(n+\frac{1}{4}\right) \pi}} .
$$

Then $-\frac{\phi\left(M_{i}\right)}{m\left(M_{i}\right)}$ lies between the greatest and the least of the fractions

$$
\frac{\frac{1}{\left(2 n+\frac{1}{2}\right)^{2} \pi^{2}}}{\frac{1}{8 n\left(n+\frac{1}{4}\right) \pi}}=\frac{2 n}{\left(n+\frac{1}{4}\right) \pi} \quad(n=i, i+1, \ldots, 2 i-1)
$$


that is,

$$
\frac{2 i}{\left(i+\frac{1}{4}\right) \pi}<-\frac{\Phi\left(M_{i}\right)}{m\left(M_{i}\right)}<\frac{2(2 i-1)}{\left(2 i-1+\frac{1}{4}\right) \pi}
$$

Let $i$ become infinite, then

$$
\lim _{i \rightarrow \infty} \frac{\phi\left(M_{i}\right)}{m\left(M_{i}\right)}=-\frac{2}{\pi}
$$

Thus we have a general derivative which is not zero. Therefore, $\phi(E)$ can not have a unique general derivative at $x=0$. 